\title{
A Comparison of the Structural, Electronic, Optical and Elastic Properties of Wurtzite, Zinc-Blende and Rock Salt TIN: A DFT Study
}

\author{
M. FARZAN ${ }^{a}$, S.M. Elahi ${ }^{a, *}$, H. SAlehi ${ }^{b}$ And M.R. AbOlHASSAni ${ }^{a}$ \\ ${ }^{a}$ Plasma Physics Research Center, Science and Research Branch, Islamic Azad University, Tehran, IR Iran \\ ${ }^{b}$ Department of Physics, Shahid Chamran University of Ahvaz, Golestan Blvd, Ahvaz, IR Iran
}

(Received January 29, 2016; in final form June 28, 2016)

\begin{abstract}
In this article we investigated structural, electronic, elastic, and optical properties of TlN in three phases, using full potential linear augmented plane wave method in the density functional theory frame with WIEN2k code. The calculations have been done in the generalized Perdew-Burke-Ernzerhof generalized gradient approximation, the generalized $\mathrm{Wu}-$ Cohen gradient approximation, the generalized Perdew-Burke-Ernzerhof solid gradient approximation, local density approximation, and the modified Becke-Johnson approximations. In spite of the absence of any experimental data for TIN, our results are compared with other results achieved by other different approximations which shows a good agreement with them. The band gap for TIN in wurtzite and zinc-blende are obtained to be 0.07 and $0.09 \mathrm{eV}$ within modified Becke-Johnson-local density approximation+spin-orbit approximation, respectively. The structural properties such as phase transitions, equilibrium lattice parameters, bulk modulus and its first pressure derivative were obtained using an optimization method. Moreover, we calculated quantities such as elastic constants, the Young modulus, shear modulus, the Poisson ratio, and sound velocities for longitudinal and transverse waves, the Debye temperature and the Kleinman parameters in different approximations and we show that TIN is softer than other nitrides of the III-group. The static calculations predicted that wurtzite to rock salt and zinc-blende to rock salt phase transitions occur at $14.7 \mathrm{GPa}$ and 15.8, respectively. The optical properties of TIN in three phases, calculated in generalized gradient approximation and local density approximation and imaginary part of dielectric function show that TIN in wurtzite and zinc-blende phases have semiconductor properties but rock salt phase do not show. As well as, we investigate the influence of the hydrostatic pressure on the elastic parameters and energy band structures for TIN (zinc-blende) within local density approximation.
\end{abstract}

DOI: 10.12693 /APhysPolA.130.758

PACS/topics: 71.15.Mb, 71.20.-b, 78.20.Ci, 62.20.Dc

\section{Introduction}

In the last years, the III-group nitrides have been investigated widely because of their high temperature stability, very short bond lengths, low compressibility, and high thermal conductivity. These materials also have excellent properties such as wide band gaps and strong bond strength, therefore they can be used for violet, blue, and green light emitting apparatuses and high temperature transistors [1-3].

However, up to our knowledge, thallium nitride (TIN) very little has been investigated. The band gap of the IIIgroup nitrides decreases from top to the bottom in element table so we expect that TIN has a small or even negative energy gap and shows a semi-metallic behavior [4]. So combinations of $\mathrm{Tl}$ with wide-gap III-nitrides yield interesting ternary alloys such as $\mathrm{Al}_{1-x} \mathrm{Tl}_{x} \mathrm{~N}[5]$ and $\mathrm{Ga}_{1-x} \mathrm{Tl}_{x} \mathrm{~N}$ [6] with great potential for infrared optical apparatuses. Experimental synthesis of TIN has not yet been reported. However, by total energy calculations from first-principles, the wurtzite phase has been found to be the ground state phase of TIN $[7,8]$. The electronic

*corresponding author; e-mail:

smohammad_elahi@srbiau.ac.ir structure and some of optical properties of wurtzite (WZ) and zinc-blende (ZB) TIN by da Silva et al. by using QP model and local density approximation (LDA) have been investigated [9].

Recently, first-principle computations based on density functional theory (DFT) have developed the fundamental part of materials investigations. The density functional theory full potential linear augmented plane wave (FPLAPW) method has been broadly recognized as a powerful method for computational solid-state researches. The calculation of various properties such as the structural, elastic, thermodynamical, optical and electronic properties for several compounds has been done by using the DFT method. These calculations provide a comprehensive knowledge about the different properties of materials and the chance to design new compounds for special applications [10]. Although there are three different phases of TIN, but no any complete study comparing TIN in these three phases has been performed. In this work, we investigated structural, electronic, elastic, and the optical properties of TIN in three phases. The calculation method was FP-LAPW with various approximations. Exchange-correlation functional and corresponding potential have an outstanding role in DFT based total energy calculations. The calculations were done using LDA, generalized gradient approximation (GGA) with 
spin-orbit (SO) and modified Becke--Johnson (mBJ) for exchange correlation functional.

\section{Computational approach}

In this work, we were applying FP-LAPW approach within the DFT framework, to obtain the structural, phase transition, elastic, thermodynamical, optical and electronic properties of TIN in three phases by using WIEN2k code [11]. We were applying to our calculation different approximations such as LDA, GGA: PerdewBurke-Ernzerhof (PBE) [12], Wu-Cohen (WC) [13], Perdew-Burke-Ernzerhof solid (PBEsol) [14], and mBJGGA or mBJ-LDA [15]. The wave functions cut-off magnitude was chosen to be $R_{\mathrm{MT}} K_{\max }=8.5$ for wurtzite and $R_{\mathrm{MT}} K_{\max }=8.0$ for cubic phase (ZB and $\mathrm{RS}$ ), in the interstitial spaces, where $R_{\mathrm{MT}}$ denoted the smallest atomic muffin-tin sphere radius and $K_{\max }$ denoted the largest $\boldsymbol{K}$ vector in the plane wave extension. The valence wave functions inside the muffin-tin spheres were expanded up to $l_{\max }=10$, while the charge density was Fourier expanded up to $G_{\max }=12$ (a.u. $)^{-1}$. The self-consistent calculations are considered to be converged when the total energy of the system is fixed within $10^{-4} \mathrm{Ry}$. The integrals over the Brillouin zone are $1000 k$-points in the irre- ducible Brillouin zone, using the Monkhorst-Pack special $k$-points method [16]. The energy dividing the valence state from the core state was set as $-6.0 \mathrm{Ry}$. In the calculations [Xe] $6 s^{2} 4 f^{14} 5 d^{10} 6 p^{1}$ and [He] $2 s^{2} 2 p^{3}$ states are considered as valence electrons for $\mathrm{Tl}$ and $\mathrm{N}$, respectively.

\section{Results and discussion}

\subsection{Structural properties}

To appraise the structural properties of TIN in three phases, the total energies are assessed for various volumes environs of the equilibrium cell volume $V_{0}$. The gained total energy is fitted to the Murnaghan equation of state [17] to assess the structural properties such as equilibrium lattice constant $a$ and $c$, the bulk modulus $B$ and its first pressure derivative $B^{\prime}$ and $E_{0}$. These equilibrium parameters are calculated using GGA (PBE, WC, and PBEsol) and LDA approximations, which are listed in Table I. Since there are not any experimental data for TIN, we compared our results with other theoretical results. According to Table I, we see that our results are inconsistent with the other results. Also, according to Table I, we can see that ground state of TIN must be in WZ phase like other the III-group nitrides because $e_{0}$ of wurtzite phase is less than other phase.

\section{TABLE I}

Calculated lattice constant, $a_{0}[\AA]$ and $c[\AA]$, bulk modulus, $B_{0}$, and its pressure derivative, $B_{0}^{\prime}$ for TIN in three phases using different approximations.

\begin{tabular}{|c|c|c|c|c|c|}
\hline Thallium nitride & $a_{0}[\AA]$ & $c[\AA]$ & $b_{0}[\mathrm{GPa}]$ & $b_{0}^{\prime}$ & $e_{0}[\mathrm{Ry}]$ \\
\hline \multicolumn{6}{|c|}{ wurtzite } \\
\hline GGA (PBE) & 3.7398 & 6.0764 & 93.85 & 4.3319 & -81374.461548 \\
\hline GGA (WC) & 3.6840 & 5.9718 & 107.71 & 4.4315 & -81368.891968 \\
\hline GGA (PBEsol) & 3.6806 & 5.9644 & 107.97 & 4.4331 & -81351.716098 \\
\hline LDA & 3.6461 & 5.8987 & 117.88 & 4.4851 & -81328.973580 \\
\hline other works & $\begin{array}{l}3.747^{a}, 3.746^{b} \\
3.766^{c}, 3.598^{d}\end{array}$ & $\begin{array}{l}6.079^{a}, 6.014^{b} \\
5.768^{c}, 5.937^{d}\end{array}$ & $85^{a}, 113^{b}, 121^{d}$ & $4.4^{a}, 4.2^{b}$ & - \\
\hline \multicolumn{6}{|c|}{ Zinc-blende } \\
\hline GGA (PBE) & 5.2723 & - & 94.06 & 4.3038 & -40687.232557 \\
\hline GGA (WC) & 5.1961 & - & 106.73 & 4.5417 & -40684.448502 \\
\hline GGA (PBEsol) & 5.1912 & - & 106.03 & 4.6325 & -40675.860657 \\
\hline LDA & 5.1415 & - & 116.61 & 4.6386 & -40664.490043 \\
\hline other works & $5.129^{e}, 4.882^{f}, 5.055^{g}$ & - & $114.9^{e}, 141^{f}, 128^{g}$ & - & - \\
\hline \multicolumn{6}{|c|}{ Rock salt } \\
\hline GGA (PBE) & 4.9563 & - & 116.04 & 4.3192 & -40687.19363 \\
\hline GGA (WC) & 4.8812 & - & 137.46 & 4.2564 & -40684.421728 \\
\hline GGA (PBEsol) & 4.8737 & - & 137.18 & 4.3034 & -40675.833034 \\
\hline LDA & 4.8312 & - & 146.71 & 4.4092 & -40664.463826 \\
\hline Ref. [19] & 4.856 & - & 112.2 & - & - \\
\hline
\end{tabular}

\subsection{Phase transformations}

Commonly, the phase equilibrium transition pressure is acquired by calculating the total energy versus the volume $(E-V)$ curves of the two phases and then obtaining their common tangent. In zero temperature, we have calculated the enthalpy $(H=E+P V)$ of TIN corresponding to the transitions WZ to rock salt (RS) and $\mathrm{ZB}$ to RS structures. It was found that the transi- 
tion pressure of TIN from WZ to RS phase was 14.7, 10.52, 10.7, and $10 \mathrm{GPa}$, and from $\mathrm{ZB}$ to $\mathrm{RS}$ to be 15.815, 10.93, 11.6 and 11.05 GPa, using GGA (PBE), GGA (WC), GGA (PBEsol) and LDA approximations, respectively. The amounts of calculating phase transition pressures, listed in Table II, are consistent with others results. It is found that the phase transition pressures decrease with the increase of the atomic radius of the III-group elements. The transition pressure depends on the following factors: (a) the bulk modulus $B_{0}$ (the larger $B_{0}$, the larger $P_{\mathrm{t}}$ ) and $(\mathrm{b})$ the difference in equilibrium volumes $\Delta V_{0}$ for different phases (the larger $\Delta V_{0}$, the smaller $P_{\mathrm{t}}$ ) [18]. According to Table II, our results in various approximations are less than the values obtained by Shi et al. [19], which indicate that our results are more favorable than their results because as the atomic radius increases the pressure at which phase transition occurs should decrease [20].

\section{TABLE II}

Phase transition pressure [GPa] for TIN.

\begin{tabular}{c|c|c}
\hline \hline TIN & WZ $\rightarrow$ RS & ZB $\rightarrow$ RS \\
\hline GGA (PBE) & 14.7 & 15.815 \\
GGA (WC) & 10.52 & 10.93 \\
GGA (PBEsol) & 10.7 & 11.6 \\
LDA & 10 & 11.05 \\
other works $^{a}$ & $19.2^{a}$ & $14.6^{b}$ \\
\hline
\end{tabular}

${ }^{a}$ Ref. [19], ${ }^{b}$ Ref. [36].

\subsection{Elastic properties}

Elastic properties have an important role in obtaining beneficial information about the anisotropic nature of bindings, structural stability and binding properties among adjacent atomic planes [21]. Hence, we investigate the stability of TIN in WZ, ZB, and RS phases using four approximations. These elastic constants are calculated by cubic-elastic and hex-elastic as interfaced to the WIEN2k code [22].

\subsubsection{Elastic constant in $W Z$ phase}

Only five independent elastic constants $\left(C_{11}, C_{12}, C_{13}\right.$, $C_{33}$, and $\left.C_{55}\right)$ are needed to be calculated for hexagonal structures. By using the calculated elastic constants, other structural properties such as bulk $(B)$, shear $(S)$ and Youngs $(Y)$ moduli and the dimensionless Poisson ratio $\nu(B S E \nu)$ are estimated, based on the Voigt, Reuss, and Hill approximations [23-25].

For hexagonal structures, the elastic parameters are given by following equations:

$$
\begin{aligned}
& b_{\mathrm{V}}=\frac{1}{9}\left(2 c_{11}+c_{33}\right)+\frac{2}{9}\left(c_{12}+2 c_{13}\right), \\
& b_{\mathrm{R}}=\frac{1}{\left(2 S_{11}+S_{33}\right)+2\left(2 S_{12}+2 S_{13}\right)},
\end{aligned}
$$

$$
\begin{aligned}
b_{\mathrm{H}} & =\frac{b_{\mathrm{V}}+b_{\mathrm{R}}}{2} \\
S_{\mathrm{V}} & =\frac{1}{15}\left(2 c_{11}+c_{33}-2 c_{13}-c_{12}\right)+\frac{1}{5}\left(2 c_{55}+c_{66}\right) \\
c_{66} & =\frac{c_{11}-c_{12}}{2}, \\
S_{\mathrm{R}} & =\frac{15}{4\left(2 S_{12}+S_{33}\right)-4\left(S_{12}+2 S_{13}\right)+3\left(2 S_{55}+S_{66}\right)} \\
S_{\mathrm{H}} & =\frac{S_{\mathrm{V}}+S_{\mathrm{R}}}{2}, \\
Y & =\frac{9 B S}{3 B+S}, \\
V & =\frac{3 B-2 S}{2(3 B+S)}
\end{aligned}
$$

where $C_{i j}$ and $S_{i j}$ are the elastic constants and elastic compliances, respectively.

Since Voigt and Reuss approximations represent the upper and lower limits of BSE $\nu$ properties [25], therefore thermodynamical properties such as average sound velocity $\left(v_{m}\right)$ which consists of the longitudinal $\left(v_{1}\right)$ and transversal $\left(v_{\mathrm{t}}\right)$ sound velocities and Debye temperature $\left(\theta_{\mathrm{D}}\right)$, are calculated using Hill approximation [25]:

$$
\begin{aligned}
& v_{\mathrm{l}}=\left(\frac{3 B_{\mathrm{H}}+4 S_{\mathrm{H}}}{3 \rho}\right)^{\frac{1}{2}} \text { and } v_{\mathrm{t}}=\left(\frac{S_{\mathrm{H}}}{\rho}\right)^{\frac{1}{2}}, \\
& v_{m}=\left[\frac{1}{3}\left(\frac{2}{v_{\mathrm{t}}^{3}}+\frac{1}{v_{\mathrm{l}}^{3}}\right)\right]^{\frac{-1}{3}} \\
& \text { and } \theta_{\mathrm{D}}=\frac{h}{k_{\mathrm{B}}}\left(\frac{3}{4 \pi V_{\mathrm{a}}}\right)^{\frac{1}{3}} v_{m},
\end{aligned}
$$

where $h$ is the Planck constant, $k_{\mathrm{B}}$ is the Boltzmann constant, $V_{\mathrm{a}}$ is the atomic volume and $\rho$ is mass density of material.

Using first-principle calculations and calculated elastic constants, it is possible to evaluate technological important properties such as stiffness, hardness, brittle/ductility and the type of bonds for crystal structures.

A material behaves as a brittle (ductile) if the $\frac{b}{S}$ ratio is less (more) than 1.75 [26]. The sign of the Cauchy pressure $\left(C_{12}-C_{55}\right)$ can be used to predict the type of bonds. When that, the Cauchy pressure is negative (positive), covalent (ionic) bonds are dominating in materials [27]. As well as, the value of the Poisson ratio can be used to predict the type of bonds. Stiffness is the resistance to deformation forces. The Young modulus is ratio of stress and strain, and it is representative of the stiffness. It says that the value of the Young modulus $(Y)$ is greater, the material is the stiffer. Hardness is relevant to how much the material is resistant to the shape changes. There are two representatives for it: (1) bulk modulus, which is relevant to the resistance against the volume changes and (2) shear modulus, which is relevant to the resistance against the reversible deformations. Hence, it is clear that shear modulus can be a better predictor for hardness [26]. Elastic constants obtained for TIN are summarized in Table III, and 
TABLE III

Elastic constant, bulk modulus $(B)$, shear modulus $(G)$, the Young modulus $(Y)$ and the Poisson ratio $(\nu)$ of TIN (WZ) within different approximations for wurtzite.

\begin{tabular}{c|c|c|c|c|c}
\hline \hline TIN $($ Wurtzite) & GGA (PBE) & GGA (WC) & GGA (PBEsol) & LDA & Other works \\
\hline$C_{11}[\mathrm{GPa}]$ & 144.9717 & 159.7167 & 161.3356 & 175.6846 & $139^{a}, 162^{b}$ \\
$C_{12}[\mathrm{GPa}]$ & 72.5384 & 89.8642 & 89.8624 & 100.8482 & $70^{a}, 77^{b}$ \\
$C_{13}[\mathrm{GPa}]$ & 54.9481 & 73.0464 & 73.2738 & 81.0707 & $50^{a}, 58^{b}$ \\
\hline$C_{33}[\mathrm{GPa}]$ & 153.7822 & 172.0633 & 172.6709 & 184.4474 & $149^{a}, 175^{b}$ \\
$C_{55}[\mathrm{GPa}]$ & 31.8582 & 31.0423 & 31.5602 & 32.7761 & $31^{a}, 36^{b}$ \\
$B_{\mathrm{V}}[\mathrm{GPa}]$ & 89.843 & 107.045 & 107.573 & 117.977 & $85^{a}, 98^{b}$ \\
$B_{\mathrm{R}}[\mathrm{GPa}]$ & 89.787 & 107.030 & 107.553 & 117.893 & \\
$B_{\mathrm{H}}[\mathrm{GPa}]$ & 89.815 & 107.037 & 107.563 & 117.935 & \\
$G_{\mathrm{V}}[\mathrm{GPa}]$ & 37.405 & 36.437 & 37.033 & 38.782 & $36^{a}$ \\
$G_{\mathrm{R}}[\mathrm{GPa}]$ & 36.319 & 35.312 & 35.942 & 37.546 & \\
$G_{\mathrm{H}}[\mathrm{GPa}]$ & 36.862 & 35.874 & 36.487 & 38.164 & \\
$Y_{\mathrm{V}}[\mathrm{GPa}]$ & 98.539 & 98.172 & 99.662 & 104.856 & $125^{a}$ \\
$Y_{\mathrm{R}}[\mathrm{GPa}]$ & 96.011 & 95.439 & 97.018 & 101.828 & \\
$Y_{\mathrm{H}}[\mathrm{GPa}]$ & 97.277 & 96.806 & 98.341 & 103.344 & \\
$\nu_{\mathrm{V}}$ & 0.317 & 0.347 & 0.345 & 0.351 & \\
$\nu_{\mathrm{R}}$ & 0.321 & 0.351 & 0.349 & 0.356 & \\
$\nu_{\mathrm{H}}$ & 0.319 & 0.349 & 0.347 & 0.353 & \\
$\theta_{\mathrm{D}}[\mathrm{K}]$ & 244.027 & 239.924 & 241.792 & 246.312 & $288^{a}$ \\
$V_{\mathrm{t}}[\mathrm{m} / \mathrm{s}]$ & 1929.15 & 1861.79 & 1874.83 & 1890.03 & $1924^{a}$ \\
$V_{1}[\mathrm{~m} / \mathrm{s}]$ & 3745.66 & 3868.32 & 3879.28 & 3975.15 & $3689^{a}$ \\
$V_{m}[\mathrm{~m} / \mathrm{s}]$ & 2160.22 & 2093.03 & 2107.23 & 2126.11 & $2553^{a}$ \\
\hline$a$ & \multicolumn{5}{|}{}
\end{tabular}

the results are in good agreement with the result of the other researchers. To our knowledge, there exist no any experimental and theoretical data for some physical quantities of TIN. We hope that our result provides a useful reference for future experimental and theoretical studies.

According to our results, the amount of $\frac{B_{\mathrm{H}}}{S_{\mathrm{H}}}$ for different approximations are equal to 2.44 (GGA (PBE)), 2.98 (GGA (WC)), 2.95 (GGA (PBEsol)), and 3.09 (LDA) which shows that TIN in WZ phase is a ductile material, but LDA predicts that TIN is more ductile than GGA approximations.

Our Cauchy pressure $\left(C_{12}-C_{55}>0\right)$ within GGA and LDA predicts that for TIN, ionic bonds are more dominant and the values of the Poisson ratio (see Table III) predicts that TIN exhibit ionic bonding.

The present value of the Young and shear moduli, Debye temperature, and average sound velocity are lower than other nitride of the III-group which shows that TIN is softer than other nitride of the III-group. The comparison of the Young modulus achieved by Shi [28] and our calculation, demonstrate that TIN is anticipated to be stiffer than our calculation. These differences could be due to lattice parameters from package $2 \mathrm{D}$ optimization (this package performed a convenient $2 \mathrm{D}$ structure optimization (volume and $c / a$, i.e. hexagonal space-group)). The previous analysis [29] on the elastic properties showed that $\frac{S}{b}$ ratio (the Pugh modulus) and revised Cauchy pressure $\frac{1}{e}\left(C_{12}-C_{55}\right)(Y$ - the Young

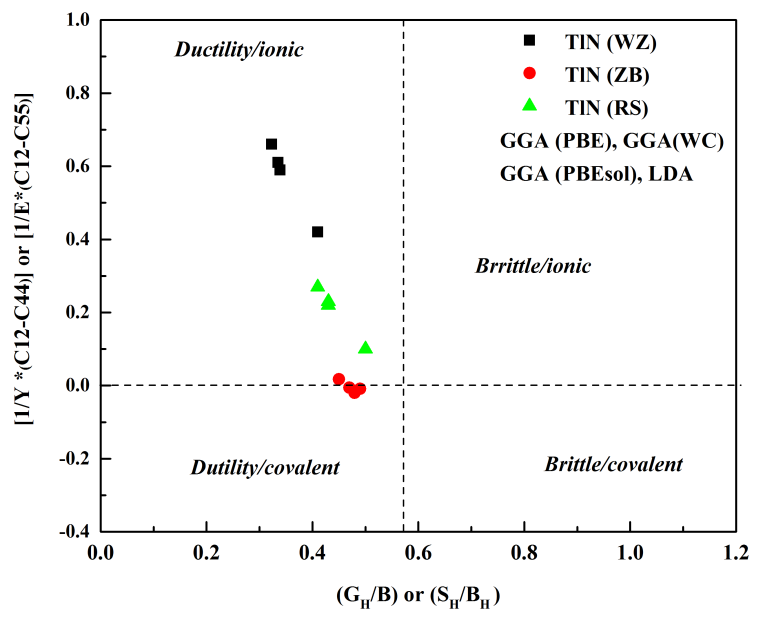

Fig. 1. The correlation between ductility/brittleness and the type of bond for TIN in three phases and different approximations.

modulus) are well correlated to a hyperbolic criterion to recognize the ductile to brittle properties for a large materials of cubic symmetry. This means that there is a correlation between ductility/brittleness property and metallic/covalent bonding [29].

The analysis by Jamal et al. [30] were applied to hexagonal compounds and they seized the right results. However, we used this analysis for TIN and found that TIN is a ductile material and has ionic bond (Fig. 1). 
3.3.2. Elastic constant in cubic phase (zinc-blende and rock salt)

There are three independent elastic constants $C_{11}, C_{12}$ and $C_{44}$ for the cubic crystals. These elastic constants were calculated by cubic-elastic method [22] as interfaced to the WIEN2k code for TIN in ZB and RS phases. The results are gathered in Tables IV and V. In these tables the previous theoretical data are also included for comparison.

TABLE IV

Elastic constant, bulk modulus $(B)$, shear modulus $(G)$, the Young modulus $(Y)$ and the Poisson ratio $(\nu)$ of TIN (ZB) within different approximations for zinc-blende.

\begin{tabular}{c|c|c|c|c|c}
\hline \hline TIN (zinc-blende) & GGA (PBE) & GGA (WC) & GGA (PBEsol) & LDA & Other works \\
\hline$C_{11}[\mathrm{GPa}]$ & 118.4151 & 130.2153 & 128.3754 & 145.1527 & $151^{a}, 194^{b}, 129^{c}$ \\
$C_{12}[\mathrm{GPa}]$ & 81.1312 & 94.1365 & 92.0789 & 107.8336 & $110^{a}, 115^{b}, 85^{c}$ \\
$C_{44}[\mathrm{GPa}]$ & 82.3647 & 95.0413 & 95.4501 & 104.8249 & $64^{a}, 103^{b}, 64^{c}$ \\
$B[\mathrm{GPa}]$ & 93.559 & 106.162 & 104.177 & 120.273 & $124^{a}, 99^{c}$ \\
$G_{\mathrm{V}}[\mathrm{GPa}]$ & 56.874 & 64.239 & 64.529 & 70.357 & \\
$G_{\mathrm{R}}[\mathrm{GPa}]$ & 34.792 & 35.104 & 35.302 & 36.818 & \\
$G_{\mathrm{H}}[\mathrm{GPa}]$ & 45.833 & 49.671 & 49.915 & 53.587 & \\
$Y[\mathrm{GPa}]$ & 141.873 & 160.370 & 160.457 & 176.629 & \\
$V_{\mathrm{t}}[\mathrm{m} / \mathrm{s}]$ & 2151.84 & 2191.79 & 2194.03 & 2240.7 & \\
$V_{1}[\mathrm{~m} / \mathrm{s}]$ & 3952.98 & 4083.17 & 4057.72 & 4238.29 & \\
$V_{m}[\mathrm{~m} / \mathrm{s}]$ & 2400.38 & 2447.41 & 2448.65 & 2504.74 & \\
$\theta_{\mathrm{D}}[\mathrm{K}]$ & 2710.096 & 280.46 & 280.867 & 290.08 & \\
melt. temp. $[\mathrm{K}]$ & $1252.83 \pm 300$ & $1322.57 \pm 300$ & $1311.69 \pm 300$ & $1410.85 \pm 300$ & \\
$\nu$ & 0.247 & 0.248 & 0.243 & 0.255 & \\
$\xi$ & 0.774 & 0.803 & 0.799 & 0.818 & \\
$A$ & 4.418 & 5.268 & 5.259 & 5.618 & \\
$\lambda$ & 55.537 & 63.230 & 61.035 & 73.242 & \\
$\mu$ & 56.886 & 64.250 & 64.544 & 70.37 & \\
$C^{\prime}$ & 18.642 & 18.039 & 18.148 & 18.659 & \\
$C^{\prime \prime}$ & -1.233 & -0.905 & -3.371 & 3.008 & \\
\hline
\end{tabular}

${ }^{a}$ Ref. [36], ${ }^{b}$ Ref. [37], ${ }^{c}$ Ref. [38].

In order to ensure the reliability of our calculations, the following well-known Born elastic stability criteria [30] for the cubic systems [31-35] are surveyed for our calculated elastic constants:

$$
\begin{aligned}
& C_{11}-C_{12}>0, \quad c_{11}+2 C_{12}>0, \quad c_{44}>0(11), \\
& C_{11}^{2}-C_{12}^{2}>0, \quad c_{11}>0 .
\end{aligned}
$$

The obtained elastic constants of TIN (ZB and RS) satisfy the above stability conditions, displaying that they are elastically stable in ZB and RS phases. Moreover, the elastic constants also satisfy the cubic stability condition, i.e. $C_{12}<B$ and $C_{11}>B$. So, the values of elastic constants are reliable for $\mathrm{TIN}$ in $\mathrm{ZB}$ and $\mathrm{RS}$ phases.

By using the calculated elastic constants, other structural properties such as bulk modulus $(B)$, the Voigt shear modulus $\left(G_{\mathrm{V}}\right)$, the Young modulus $(Y)$, shear constant $\left(C^{\prime}\right)$, the Cauchy pressure $\left(C^{\prime \prime}\right)$, the Poisson ratio $(\nu)$, the Kleinman parameter $(\xi)$, the Reuss shear modulus $\left(G_{\mathrm{R}}\right)$, the Hill shear modulus $\left(G_{\mathrm{H}}\right)$, anisotropy constant $(A)$, and the Lame coefficients $(\lambda$ and $\mu)$ are calculated.

$$
\begin{aligned}
& B=\frac{1}{3}\left(c_{11}+2 c_{12}\right), \\
& g_{\mathrm{V}}=\frac{1}{5}\left(3 c_{44}+c_{11}-c_{12}\right), \\
& Y=\frac{9 B g_{\mathrm{V}}}{3 B+g_{\mathrm{V}}}, \\
& c^{\prime}=\frac{1}{2}\left(c_{11}-c_{12}\right), \\
& C^{\prime \prime}=C_{12}-C_{44}, \\
& v=-1+\frac{Y}{2 G_{\mathrm{V}}}=\frac{3 B-Y}{6 B}=\frac{1}{2}-\frac{Y}{6 B}, \\
& \xi=\frac{C_{11}+8 C_{12}}{7 C_{11}+2 C_{12}}, \\
& G_{\mathrm{R}}=\frac{5\left(C_{11}-C_{12}\right) C_{44}}{4 C_{44}+3\left(C_{11}-C_{12}\right)}, \\
& G_{\mathrm{H}}=\frac{G_{\mathrm{V}}+G_{\mathrm{R}}}{2},
\end{aligned}
$$


TABLE V

Elastic constant, bulk modulus $(B)$, shear modulus $(G)$, the Young modulus $(Y)$ and the Poisson ratio $(\nu)$ of TIN (RS) within different approximations for rock salt.

\begin{tabular}{c|c|c|c|c|c}
\hline \hline TIN (rock salt) & GGA (PBE) & GGA (WC) & GGA (PBEsol) & LDA & Ref. [19] \\
\hline$C_{11}[\mathrm{GPa}]$ & 165.4318 & 192.3975 & 189.8594 & 200.1031 & 155 \\
$C_{12}[\mathrm{GPa}]$ & 85.5868 & 104.9100 & 104.5691 & 113.4406 & 91 \\
$C_{44}[\mathrm{GPa}]$ & 70.4411 & 70.1324 & 69.2544 & 70.4957 & 71 \\
$B[\mathrm{GPa}]$ & 112.201 & 134.072 & 132.999 & 142.328 & 112 \\
$G_{\mathrm{V}}[\mathrm{GPa}]$ & 58.233 & 59.576 & 58.61 & 59.629 & \\
$G_{\mathrm{R}}[\mathrm{GPa}]$ & 53.945 & 56.499 & 55.421 & 56.362 & \\
$G_{\mathrm{H}}[\mathrm{GPa}]$ & 56.089 & 58.037 & 57.015 & 57.995 & \\
$Y[\mathrm{GPa}]$ & 148.933 & 155.670 & 153.309 & 156.966 & \\
$V_{\mathrm{t}}[\mathrm{m} / \mathrm{s}$ & 2168.05 & 2157.08 & 2133.09 & 2123.29 & \\
$V_{1}[\mathrm{~m} / \mathrm{s}]$ & 3958.55 & 4117.40 & 4084.20 & 4132.23 & \\
$V_{m}[\mathrm{~m} / \mathrm{s}]$ & 2417.34 & 2412.75 & 2386.41 & 2377.97 & \\
$\theta_{\mathrm{D}}[\mathrm{K}]$ & 290.565 & 294.326 & 291.56 & 293.081 & \\
$\mathrm{melt.} \mathrm{temp.}[\mathrm{K}]$ & $1530.70 \pm 300$ & $1690.07 \pm 300$ & $1675.07 \pm 300$ & $1735.61 \pm 300$ & \\
$\nu$ & 0.278 & 0.306 & 0.307 & 0.316 & \\
$\xi$ & 0.639 & 0.663 & 0.667 & 0.680 & \\
$A$ & 1.764 & 1.603 & 1.624 & 1.627 & \\
$\lambda$ & 72.966 & 94.005 & 93.292 & 102.421 & \\
$\mu$ & 58.268 & 59.598 & 58.649 & 59.637 & \\
$C^{\prime}$ & 39.922 & 43.744 & 42.685 & 43.331 & \\
$C^{\prime \prime}$ & 15.146 & 34.778 & 35.314 & 42.945 &
\end{tabular}

$$
\begin{aligned}
A & =\frac{2 C_{44}}{C_{11}-C_{12}}, \\
\lambda & =\frac{Y v}{(1+v)(1-2 v)} \text { and } \mu=\frac{Y}{2(1+v)} .
\end{aligned}
$$

Also, average velocity $\left(v_{m}\right)$, longitudinal velocity $\left(v_{1}\right)$, transverse velocity $\left(v_{\mathrm{t}}\right)$, and the Debye temperature $\left(\theta_{\mathrm{D}}\right)$ can also be attained by elastic constants and mass density. The terms are as follows:

$$
\begin{aligned}
& v_{\mathrm{l}}=\left(\frac{3 B_{\mathrm{H}}+4 S_{\mathrm{H}}}{3 \rho}\right)^{\frac{1}{2}}, v_{\mathrm{t}}=\left(\frac{S_{\mathrm{H}}}{\rho}\right)^{\frac{1}{2}}, \\
& v_{m}=\left[\frac{1}{3}\left(\frac{2}{v_{\mathrm{t}}^{3}}+\frac{1}{v_{\mathrm{l}}^{3}}\right)\right]^{\frac{-1}{3}}, \\
& \theta_{\mathrm{D}}=\frac{h}{k_{\mathrm{B}}}\left(\frac{3}{4 \pi V_{\mathrm{a}}}\right)^{\frac{1}{3}} v_{m},
\end{aligned}
$$

where $h$ is the Planck constant and $n, N, \rho, M$, and $k_{\mathrm{B}}$ are the number of atoms in the molecule, the Avogadro number, mass density, molecular weight and the Boltzmann constant, respectively.

The bulk modulus (B) and the Hill shear modulus $\left(G_{\mathrm{H}}\right)$ are significant parameters in recognizing the physical properties of materials. Bulk modulus $(B)$, the Voigt shear modulus $\left(G_{\mathrm{V}}\right)$, the Reuss shear modulus $\left(G_{\mathrm{R}}\right)$, shear modulus $\left(G_{\mathrm{H}}\right)$, the Young modulus $(Y)$ and the Poisson ratio of TIN in $\mathrm{ZB}$ and $\mathrm{RS}$ phases have been calculated using GGA (PBE), GGA (WC), GGA (PBEsol), and LDA approximations, which are summarized in Tables IV and V.
According to Table IV, we see that our results with LDA approximation (full potential) are in agreement with other results within the LDA approximation (pseudopotentials) [36-38] but our results with GGA approximations do not agree with other results. In GGA approximations, we can see that value of the $C^{\prime \prime}$ is negative $\left(C^{\prime \prime}<0\right)$ and it shows that covalent bond is more dominating, but in LDA approximation the value of $C^{\prime \prime}$ is positive $\left(C^{\prime \prime}>0\right)$ and it shows that ionic bond is more dominating which is in agreement with other results. But the value of $C^{\prime \prime}$, using any of four approximations, is nearly zero. So we can conclude that bonding of TIN in $\mathrm{ZB}$ phase is covalent-ionic. In addition to $C^{\prime \prime}$, we can consider the Poisson ratio $(\nu)$, the value of $\nu$ is much less than 0.25 (around 0.1) for a typical covalent compound, while it is nearly 0.25 or more for a typical ionic compound. According to Table IV, the values of $\nu$ using any of four approximations are around 0.25 , so we can conclude that bond of $\mathrm{TIN}$ in $\mathrm{ZB}$ phase is covalent-ionic (similar to $C^{\prime \prime}$ ).

According to our results, the amount of $\frac{b}{G_{\mathrm{H}}}$ for different approximations is equal to 2.04 (GGA (PBE)), 2.13 (GGA (WC)), 2.08 (GGA (PBEsol)), and 2.24 (LDA) which shows that TIN in ZB phase is a ductile material, but LDA predicts that it is more ductile than GGA approximations. Also, by considering the Pugh modulus [29] in Fig. 1, one can defer that TIN is a ductile material and bonds in TIN are covalent-ionic. The achieved value of the Young moduli, Debye temperature, and average sound velocity are lower than those of other nitrides 
of the III-group which shows that TIN in ZB phase is softer than other nitride of the III-group.

The dimensionless Kleinman parameter can be generally between 0 and $1(0 \leq \zeta \leq 1)$. The lower limit corresponds to the minimized bond bending term, while the upper limit corresponds to the minimized bond stretching term, as defined by Kleinman [39]. Such a large calculated value of the Kleinman parameter (see Table IV) predicts that bonding nature in TIN (ZB) is dominated by the bond stretching term in comparison to the bond bending term.

The Lamé moduli are calculated using Eq. (23) for TIN. The results are included in Table IV. However, we have calculated the Lamé second modulus as $\mu=\frac{Y}{2(1+v)}$, our result verifies that it is nothing more than the Voigt shear modulus, viz. $\mu=G_{\mathrm{V}}$ (see Table IV). The Lamé first modulus, $\lambda$, is related to a fraction of the Young modulus. For an isotropic system one can easily show that $\lambda=C_{12}$ and $\mu=C^{\prime}=\left(C_{11}-C_{12}\right) / 2=C_{44}$ [40]. But, since the TIN is a strongly anisotropic compound $\left(A \neq 1\right.$ see Table IV) so, $\mu \neq C_{44}$ (see Table IV).

According to Table V, we see that our results achieved by LDA and GGA approximations (using full potential) are in agreement with other results (using pseudopotentials) [19]. The value of $C^{\prime \prime}$ is positive (Table $\mathrm{V}$ ) which shows that bonds in TIN (RS) are ionic. According to Table $\mathrm{V}$, we see that values of $\nu$ in four approximations are greater than 0.25 , so we can conclude that the bonds of TIN in RS phase are ionic ( $\left.\operatorname{similar} C^{\prime \prime}\right)$.

According to our results, the amount of $\frac{b}{G_{\mathrm{H}}}$ for different approximations is equal to 2.0 (GGA (PBE)), 2.31 (GGA (WC)), 2.33 (GGA (PBEsol)), and 2.45 (LDA) which shows that TIN in RS phase is a ductile material. Also, considering the Pugh modulus [29] in Fig. 1 indicates that TIN in RS phase is a ductile material and its bonds are ionic. The achieved values of the Young moduli, the Debye temperature, and average sound velocity are lower than other nitrides of the III-group, which shows that TIN in RS phase is softer than other nitrides of the III-group. The calculated large value for the Kleinman parameter (see Table $\mathrm{V}$ ) predicts that bonding nature in TIN (RS) is dominated by the bond stretching term in comparison to the bond bending term. The value of $A$ parameter is around one (see Table V), that shows TIN in RS phase is not strongly anisotropic and is almost isotropic, especially in GGA (PBE) approximation because $\mu \cong$ $C_{44}$ (see Table V).

Figure 2 shows the variations of elastic constants $C_{i j}$ and their aggregate bulk modulus with a hydrostatic pressure for ZB TIN. One obviously observes a linear dependence in all curves of this compound. Our results for the pressure derivatives $\frac{\partial C_{11}}{\partial P}, \frac{\partial C_{21}}{\partial P}, \frac{\partial C_{44}}{\partial P}$, and $\frac{\partial B}{\partial P}$ of ZB TIN are listed in Table VI. It is easy to observe that the elastic constants $C_{11}, C_{21}$ and $C_{44}$, as well as bulk modulus $B$ exhibit a linearly increasing trend as pres- sure enhances. Our results are in agreement with other results, but the values of $\frac{\partial C_{44}}{\partial P}$ we have calculated, differ with other results (however, our $\frac{\partial C_{44}}{\partial P}$ is similar to other nitrides of the III-group) [36].

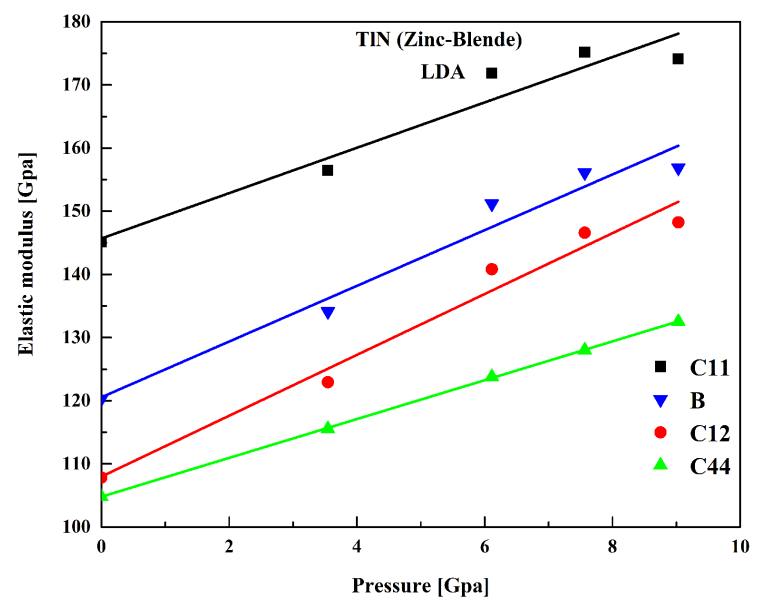

Fig. 2. The elastic constant versus pressure for TIN (ZB) within LDA approximation.

TABLE VI

Calculated pressure derivatives of the elastic moduli of TIN (ZB) within LDA approximation.

\begin{tabular}{c|c|c|c|c}
\hline \hline TIN (zinc-blende) & $\partial C_{11} / \partial P$ & $\partial C_{21} / \partial P$ & $\partial C_{44} / \partial P$ & $\partial B / \partial P$ \\
\hline present work & 3.590 & 4.818 & 3.07 & 4.409 \\
Ref. [36] & 3.607 & 4.875 & -1.438 & 4.453
\end{tabular}

\section{Electronic properties}

\subsection{Band structure}

Electronic properties of TIN in three phases were investigated by calculating the energy band structure. Table VII shows the calculated band gap energies of TIN using GGA (PBE), LDA, mBJ-GGA, mBJ-LDA, EngelVosko and mBJ-LDA+SO approximations along some high symmetry directions of the Brillouin zone calculated at equilibrium volume. The band gap energies of TIN in $\mathrm{WZ}$ and $\mathrm{ZB}$ phases within mBJ-LDA+SO approximation were improved in comparison with other theoretical results. We calculated a direct band gap for TIN (ZB) about $0.09 \mathrm{eV}$ and TIN (WZ) about $0.07 \mathrm{eV}$ but TIN in RS phase shows metallic behavior (Fig. 3). The effect of pressure on the band gap of TIN (ZB) was also investigated up to the first order phase transition pressure. The results are listed in Table VIII. It can be seen that the band gap of TIN (ZB) increases with increase of the pressure. As a rule, direct band gaps increase and indirect band gaps decrease with increase of the pressure [41]. 
TABLE VII

The band gap energy $[\mathrm{eV}]$ of TIN within different approximations.

\begin{tabular}{|c|c|c|c|c|c|c|c|}
\hline $\mathrm{TlN}$ & GGA (PBE) & LDA & mBJ (GGA) & mBJ (LDA) & $\begin{array}{c}\text { E. Vosko } \\
\text { (GGA-WC) }\end{array}$ & $\mathrm{mBJ}(\mathrm{LDA}+\mathrm{SO})$ & Other works \\
\hline \multicolumn{8}{|c|}{ wurtzite } \\
\hline$E_{\mathrm{g}}\left(\Gamma^{v} \rightarrow \Gamma^{c}\right)$ & 0.0 & 0.0 & 0.0 & 0.0 & 0.0 & 0.07 & $0.0^{a}$ \\
\hline \multicolumn{8}{|c|}{ zinc-blende } \\
\hline$E_{\mathrm{g}}\left(\Gamma^{v} \rightarrow \Gamma^{c}\right)$ & 0.0 & 0.0 & 0.0 & 0.0 & 0.0 & 0.09 & $0.0^{a, b}$ \\
\hline
\end{tabular}
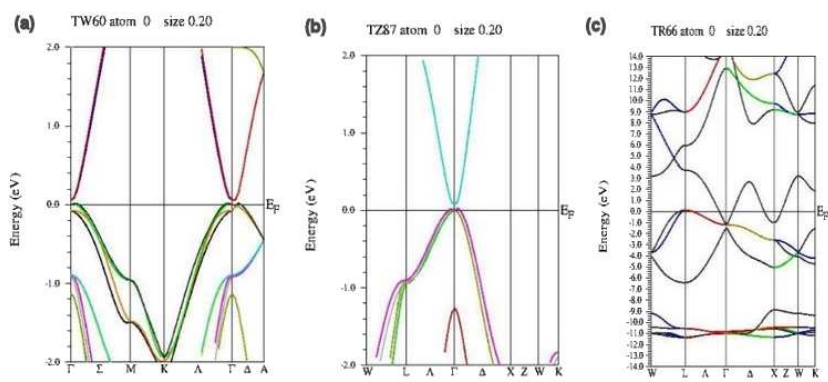

Fig. 3. The band structure of TIN - WZ (a), TIN $\mathrm{ZB}$ (b), and TIN - RS (c) within mBJ-LDA+SO.

TABLE VIII

The band gap energy $[\mathrm{eV}]$ in different pressures [GPa] for TIN (ZB) within mBJ (LDA+SO) approximation.

\begin{tabular}{c|c|c}
\hline \hline $\begin{array}{c}\text { TIN (zinc-blende) } \\
\text { pressure }[\mathrm{GPa}]\end{array}$ & $\begin{array}{c}\text { Lattice } \\
\text { constant }[\AA]\end{array}$ & $\begin{array}{c}\text { Band gap }[\mathrm{eV}] \\
\text { in mBJ (LDA+SO) } \\
\text { approximation }\end{array}$ \\
\hline$P=0.0$ & 5.1415 & 0.09 \\
$P=3.546$ & 5.094 & 0.10 \\
$P=6.114$ & 5.062 & 0.11 \\
$P=7.566$ & 5.045 & 0.112 \\
$P=9.031$ & 5.029 & 0.12
\end{tabular}

\subsection{Optical properties}

Optical calculations are performed in the random phase approximation (RPA) by using WIEN2k code. Dielectric function $\left(\varepsilon(\omega)=\varepsilon_{1}(\omega)+\mathrm{i} \varepsilon_{2}(\omega)\right)$ is a complex quantity that describes the linear response of the system to an electromagnetic radiation. The imaginary part of dielectric function is obtained by calculating momentum matrix elements between the occupied and unoccupied wave [43]:

$$
\begin{aligned}
& \operatorname{Im} \varepsilon_{\alpha \beta}^{(\text {inter })}(\omega)=\frac{h^{2} e^{2}}{\pi m^{2} \omega^{2}} \sum_{n} \int \mathrm{d} \boldsymbol{k}\left\langle\psi_{\boldsymbol{k}}^{c_{n}}\left|\boldsymbol{p}^{\alpha}\right| \psi_{\boldsymbol{k}}^{V_{n}}\right\rangle \\
& \quad \times\left\langle\psi_{\boldsymbol{k}}^{V_{n}}\left|\boldsymbol{p}^{\beta}\right| \psi_{\boldsymbol{k}}^{c_{n}}\right\rangle \delta\left(E_{\boldsymbol{k}}^{c_{n}}-E_{\boldsymbol{k}}^{V_{n}}-\omega\right),
\end{aligned}
$$

where $\boldsymbol{p}$ is the momentum matrix element between $\alpha$ and $\beta$ bands with the same crystal $\left(\psi_{\boldsymbol{k}}^{c_{n}}\right)$ and $\left(\psi_{\boldsymbol{k}}^{V_{n}}\right)$ which are the crystal wave function corresponding to the conduction and valence bands with the same crystal wave vector $\boldsymbol{k}$, respectively. The interband expansion on the corresponding real part was obtained by the Kramers-Kronig transformation

$$
\operatorname{Re} \varepsilon_{\alpha \beta}^{\text {(inter) }}(\omega)=\delta_{\alpha \beta}+\frac{2}{\pi} P \int_{0}^{\infty} \frac{\omega^{\prime} \operatorname{Im} \varepsilon_{\alpha \beta}\left(\omega^{\prime}\right)}{\left(\omega^{\prime}\right)^{2}-\omega^{2}} .
$$

Considerable optical functions like the refraction in$\operatorname{dex} n(\omega)$ and extinction coefficient $k(\omega)$ can be appraised by the following equations [44-46]:

$$
\begin{aligned}
& n(\omega)=\frac{1}{\sqrt{2}} \sqrt{\varepsilon_{1}+\sqrt{\varepsilon_{1}^{2}+\varepsilon_{2}^{2}}}, \\
& k(\omega)=\frac{1}{\sqrt{2}} \sqrt{\sqrt{\varepsilon_{1}^{2}+\varepsilon_{2}^{2}}-\varepsilon_{1} .}
\end{aligned}
$$

Optical quantities of wurtzite phase were calculated in two directions: (i) when polarized electric field is parallel to $c(E \| z)$, (ii) when polarized electrical field is perpendicular to $c(E \| x)$.

Figure 4 and 5 illustrate the real and imaginary parts of the dielectric function spectrum of TIN for a radiation up to $14 \mathrm{eV}$ within GGA (PBE) and LDA approximations. According to Fig. 4a and Fig. 5 (imaginary part of dielectric function), we see that band gap energies are very small as it is evident also in Fig. 3 for TIN in wurtzite and zinc-blende phases by using GGA (PBE) and LDA

\begin{tabular}{|c|c|c|c|c|c|}
\hline TIN & $\begin{array}{c}\text { GGA } \\
(\mathrm{PBE}) \\
(E \| x)\end{array}$ & $\begin{array}{l}\mathrm{LDA} \\
(E \| x)\end{array}$ & $\begin{array}{c}\text { GGA } \\
(\mathrm{PBE}) \\
(E \| z)\end{array}$ & $\begin{array}{l}\mathrm{LDA} \\
(E \| z)\end{array}$ & Ref. [9] \\
\hline wurtzite & 6.68 & 6.84 & 3.17 & 3.50 & $\begin{array}{l}4(E \| x), \\
3(E \| z)\end{array}$ \\
\hline zinc-blende & 6.63 & 6.84 & - & - & 8.5 \\
\hline rock salt & 3.28 & 3.57 & - & - & - \\
\hline
\end{tabular}
approximations.

TABLE IX

The main peaks position $[\mathrm{eV}]$ of the imaginary part of dielectric function for TIN in three phases.

The main peaks in the spectra of imaginary part of dielectric function (Fig. 4a and Fig. 5) are given in Table IX which are in good agreement with other reports [9]. The static dielectric constants which are extracted from the diagram of $\varepsilon_{1}(\omega)$ (Fig. $4 \mathrm{~b}$ and Fig. 5) are summarized in Table $\mathrm{X}$ and are in good agreement with previous studies [9] and we see that the static dielectric constant 

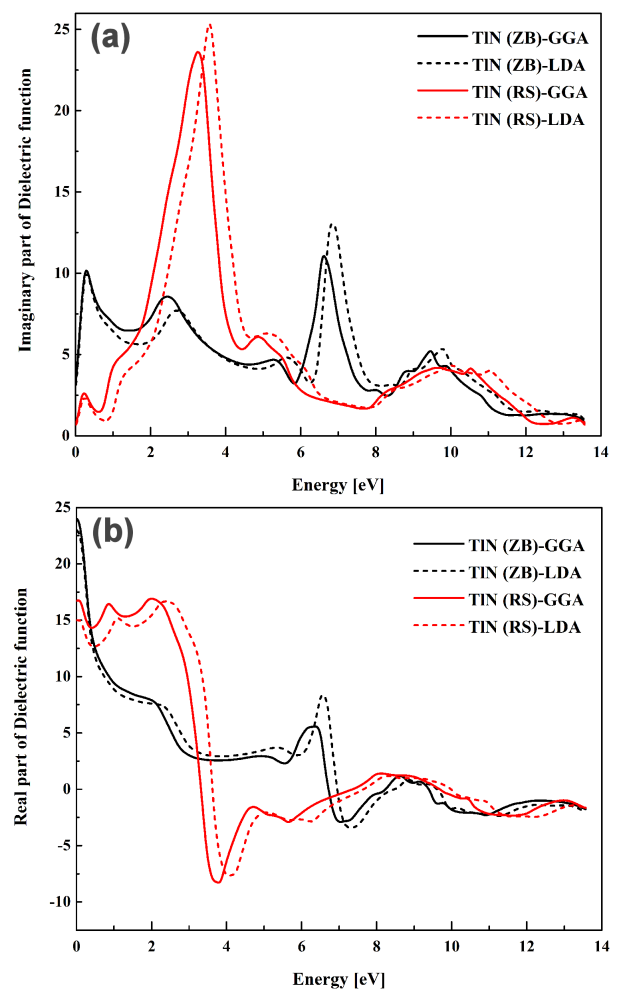

Fig. 4. The imaginary part of dielectric function (a) and the real part of dielectric function (b) versus energy for TIN in ZB and RS phases within GGA (PBE) and LDA approximations.

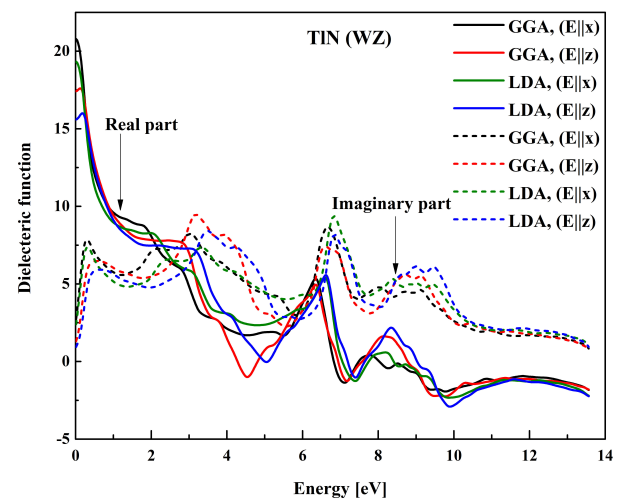

Fig. 5. The dielectric function for TIN in WZ phase within GGA (PBE) and LDA approximations in two directions $(E \| x)$ and $(E \| z)$.

TABLE X

The static dielectric constant for TIN in three phases within GGA (PBE) and LDA approximations.

\begin{tabular}{|c|c|c|c|c|c|}
\hline $\mathrm{TlN}$ & $\begin{array}{c}\text { GGA } \\
(\mathrm{PBE}) \\
(E \| x)\end{array}$ & $\begin{array}{l}\text { LDA } \\
(E \| x)\end{array}$ & $\begin{array}{c}\text { GGA } \\
(\mathrm{PBE}) \\
(E \| z)\end{array}$ & $\begin{array}{l}\text { LDA } \\
(E \| z)\end{array}$ & Ref. [9] \\
\hline wurtzite & 20.8 & 19.3 & 17 & 15.6 & $\begin{array}{c}20(E \| x), \\
22.5(E \| z)\end{array}$ \\
\hline zinc-blende & 24 & 23 & - & - & 25 \\
\hline rock salt & 16.7 & 15 & - & - & - \\
\hline
\end{tabular}

of TIN is bigger than the other III-group nitrides [47-49]. The static refraction index (Eq. (27) and Fig. 6) of TIN in GGA (PBE) and LDA approximations are given in Table XI and we see that the refraction index of TIN is bigger than the other III-group nitrides [47-49].
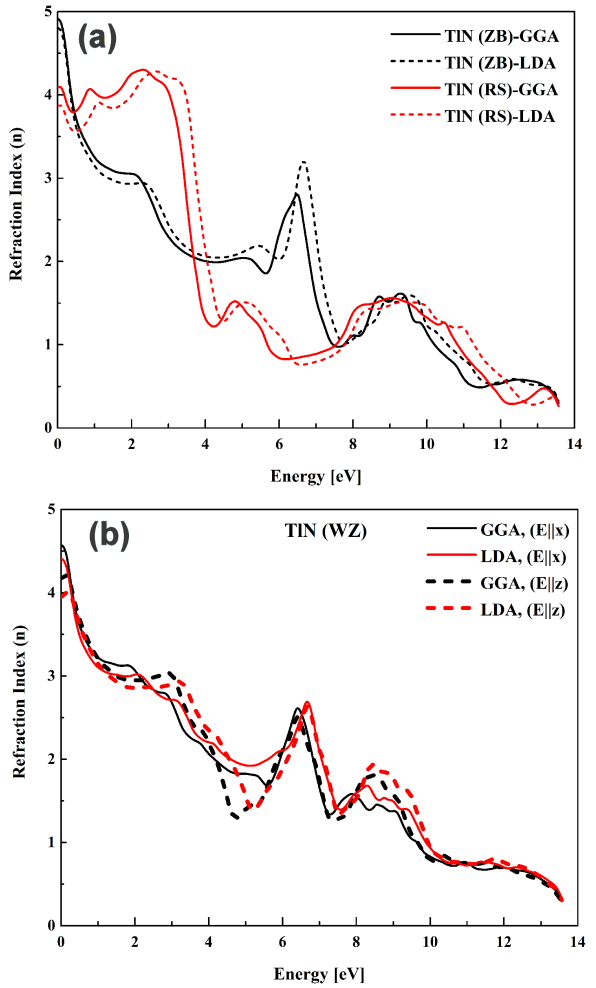

Fig. 6. The refraction index of TIN versus energy in $\mathrm{ZB}$ and RS phases (a) within GGA (PBE) and LDA approximations and WZ phase (b) within GGA (PBE) and LDA approximations in two directions $(E \| x)$ and $(E \| z)$.

TABLE XI

The static refraction for TIN in three phases within GGA (PBE) and LDA approximations.

\begin{tabular}{c|c|c|c|c}
\hline \hline TIN & $\begin{array}{c}\text { GGA } \\
(\mathrm{PBE}), \\
(E \| x)\end{array}$ & $\begin{array}{c}\text { LDA, } \\
(E \| x)\end{array}$ & $\begin{array}{c}\text { GGA } \\
(\mathrm{PBE}), \\
(E \| z)\end{array}$ & $\begin{array}{c}\text { LDA, } \\
(E \| z)\end{array}$ \\
\hline wurtzite & 4.57 & 4.4 & 4.17 & 3.95 \\
zinc-blende & 4.91 & 4.8 & - & - \\
rock salt & 4.09 & 3.87 & - & -
\end{tabular}

To check the macroscopic, microscopic, and optical properties of solids, the energy-loss function $(L(\omega))$ is one of the most important quantity. The energy-loss function is proportional to the probability of energy loss $(E)$ in a unit of length as an electron is moving through the environment and it is given by

$$
L(\omega)=\operatorname{Im}\left(\frac{-1}{\varepsilon(\omega)}\right)=\frac{\varepsilon_{2}^{2}}{\varepsilon_{1}^{2}+\varepsilon_{2}^{2}} .
$$

The main peak in the energy-loss function is known as 
plasmon peak, which indicates the excitation volume charge density in crystals. The main peaks of energy-loss function for TIN are shown in Fig. 7 and their position are indicated in Table XII.
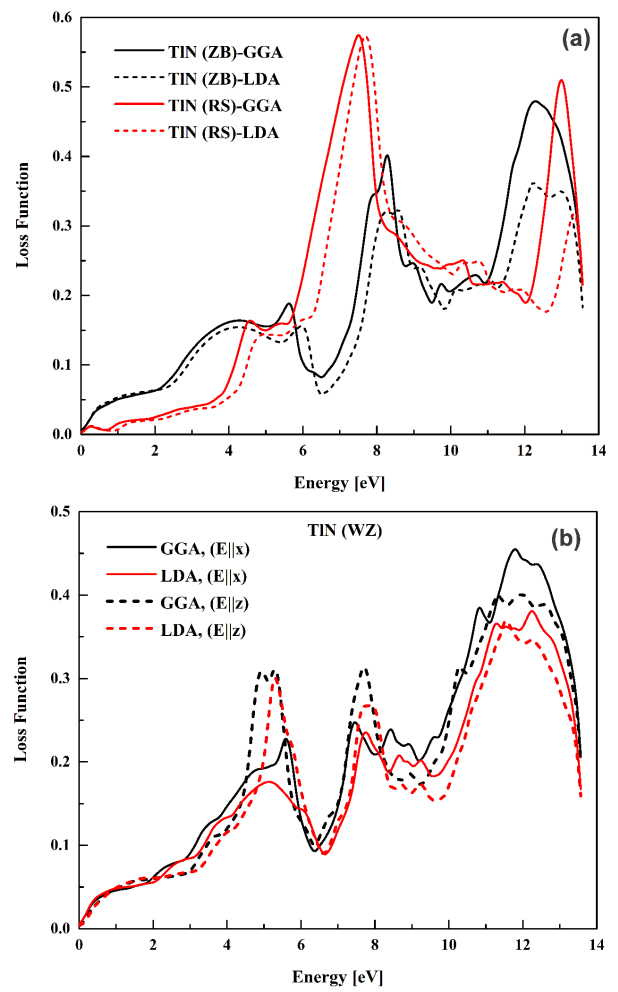

Fig. 7. The energy-loss function of TIN versus energy in ZB and RS phases (a) within GGA (PBE) and LDA approximations and WZ phase (b) within GGA (PBE) and LDA approximations and two directions $(E \| x)$ and $(E \| z)$.

TABLE XII

Value of energy $[\mathrm{eV}]$ plasmon peak for TIN in three phases within GGA (PBE) and LDA approximations.

\begin{tabular}{c|c|c|c|c}
\hline \hline TIN & $\begin{array}{c}\text { GGA } \\
(\mathrm{PBE}), \\
(E \| x)\end{array}$ & $\begin{array}{c}\text { LDA, } \\
(E \| x)\end{array}$ & $\begin{array}{c}\text { GGA } \\
(\mathrm{PBE}), \\
(E \| z)\end{array}$ & $\begin{array}{c}\text { LDA, } \\
(E \| z)\end{array}$ \\
\hline wurtzite & 11.80 & 12.26 & 11.98 & 11.55 \\
zinc-blende & 12.28 & 12.23 & - & - \\
rock salt & 7.50 & 7.69 & - & -
\end{tabular}

Two other important quantities of optical properties are absorption coefficient and real part of the optical conductivity and they are calculated by using the following relations:

$$
\begin{aligned}
& a_{i j}(\omega)=\frac{2 \omega k_{i j}(\omega)}{c}, \\
& \operatorname{Re} \sigma_{i j}(\omega)=\frac{\omega}{4 \pi} \operatorname{Im} \varepsilon_{i j}(\omega) .
\end{aligned}
$$

Absorption coefficient depends on tow quantities, extinction index and imaginary part of the dielectric function. Absorption coefficients for TIN in three different phases are shown in Fig. 8 and the maxima of absorption

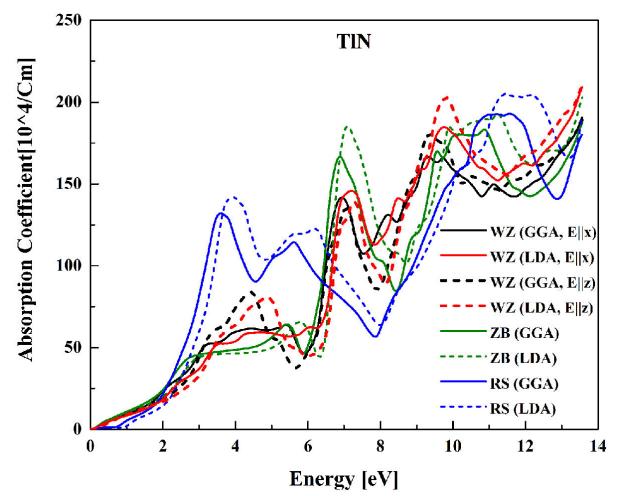

Fig. 8. The absorption coefficient of TIN in three phases within GGA (PBE) and LDA approximations and in two directions $(E \| x)$ and $(E \| z)$ for wurtzite phase.

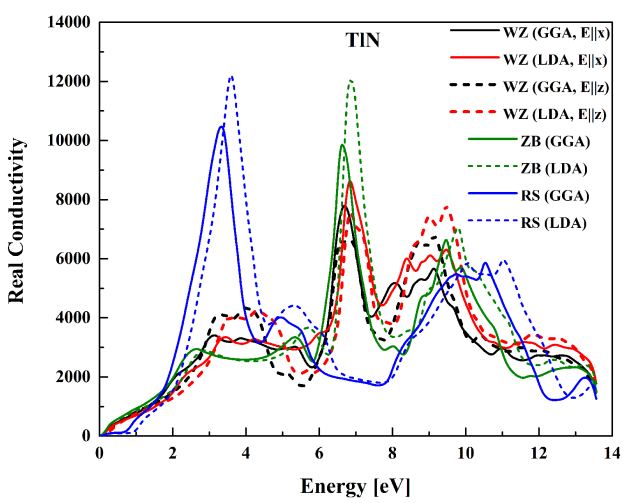

Fig. 9. The real part of optical conductivity for TIN in three phases within GGA (PBE) and LDA approximations and in two directions $(E \| x)$ and $(E \| z)$ for wurtzite phase.

coefficients can be seen in Fig. 8. Also, according to Eq. (31), we see that the real part of the optical conductivity is related to the imaginary part of dielectric function.

As it is clear in Fig. 9 the real part of the optical conductivity for TIN starts with a very small gap which indicate that the TIN has semiconductor properties (especially in two phases $\mathrm{WZ}$ and $\mathrm{ZB}$ ).

\section{Conclusion}

In summary, we have performed $a b$ initio calculations of structural, elastic, electronic, and optical properties of TIN in three different phases by using the FP-LAPW method. The results predict that $\mathrm{TIN}$ in $\mathrm{WZ}$ and $\mathrm{ZB}$ phases is semiconductor with a direct band gap while TIN in RS phase shows metallic behavior. Although TIN in three phases is ductile material, according to the Poisson ratio $\mathrm{TIN}$ in $\mathrm{WZ}$ and $\mathrm{RS}$ phases has ionic bond and in ZB phase has an ionic-covalent bond. The pressures at which the first order phase transitions occur were also estimated and compared with available data. Furthermore, the elastic constants, bulk modulus, shear modulus, the Young modulus, the Poisson ratio, Debye temperature, and sound velocities for longitudinal and 
transverse waves were calculated and they show that TIN is softer than other nitrides of the III-group. The variations of band gap energy, elastic constants and the bulk modulus of TIN (ZB) versus pressure were calculated and discussed and show that the band gap and elastic constant increase with increasing pressure. The optical parameters of TIN in three phases were also calculated and analyzed and imaginary part of the dielectric function show that TIN in WZ and ZB phases have almost semiconductor properties and $\mathrm{TIN}$ in RS phases has metallic properties. Also, real part of conductivity shows that TIN in WZ and ZB phase has semiconductor properties.

\section{References}

[1] I. Vurgaftman, J.R. Meyer, L.R. Ram-Mohan, J. Appl. Phys. 89, 5815 (2001).

[2] S.C. Jain, M. Willander, J. Narayam, R. Varayan, R. Vanoverstrateten, J. Appl. Phys. 87, 965 (2000).

[3] I. Vurgaftman, J.R. Meyer, J. Appl. Phys. 94, 3675 (2003).

[4] A. Zaoui, Mater. Sci. Eng. B 103, 258 (2003).

[5] N.S. Dantas, J.S. de Almeria, R. Ahuja, C. Persson, A. da Silva, Appl. Phys. Lett. 92, 121914 (2008).

[6] N. Saidi-Houat, A. Zaoui, A. Belabbes, M. Ferhat, Mater. Sci. Eng. B 162, 26 (2009).

[7] M. Ferhat, A. Zaoui, Appl. Phys. Lett. 88, 161902 (2006).

[8] N. Saidi-Houat, A. Zaoui, M. Ferhat, J. Phys. Condens. Matter 19, 106221 (2007).

[9] A.F. da Silva, N.S. Dantas, J.S. de Almeida, R. Ahuja, C. Persson, J. Cryst. Growth 281, 151 (2005).

[10] S.Q. Wang, H.Q. Ye, Phys. Rev. B 66, 235111 (2002).

[11] P. Blaha, K. Schwarz, G. Madsen, D. Kvasnika, K. Luitz, WIEN2k, an augmented plane wave plus local orbitals program for calculating crystal properties, Vienna, Austria 2001.

[12] J.P. Perdew, K. Burke, M. Ernzerhof, Phys. Rev. Lett. 77, 3865 (1996).

[13] Z. Wu, R.E. Cohen, Phys. Rev. B 73, 235116 (2006).

[14] J.P. Perdew, A. Ruzsinszky, G.I. Csonka, O.A. Vydrov, G.E. Scuseria, L.A. Constantin, X. Zhou, K. Burke, Phys. Rev. Lett. 100, 136406 (2008).

[15] F. Tran, P. Blaha, Phys. Rev. Lett. 102, 226401 (2009).

[16] H. Pack, J. Monkhorst, Phys. Rev. B 13, 5188 (1976).

[17] F.D. Murnaghan, PNAS 30, 244 (1944).

[18] R. Wentzcovitch, M. Cohen, P. Lam, Phys. Rev. B 36, 6058 (1987).

[19] L. Shi, Y. Duan, L.X. Qin, Chin. Phys. Lett. 27, 080505 (2010).

[20] C. Pinquier, F. Demangeot, J. Frandon, J.-C. Chervin, A. Polian, B. Couzinet, P. Munsch, O. Briot, S. Ruffenach, B. Gil, B. Maleyre, Phys. Rev. B 73, 115211 (2006).

[21] A. Bouhemadou, R. Khenata, F. Zegrar, M. Sahnoun, H. Baltache, A.H. Reshakd, Comput. Mater. Sci. 38, 263 (2006).
[22] M. Jamal, Cubic-elastic and Hex-elastic, www.wien2k.at/reg_user/, unsupported (2012).

[23] M. Voigt, Lehrbuch der Kristallphysik, Taubner, Leipzig 1928.

[24] A. Reuss, Z. Angew. Math. Mech. 9, 49 (1929).

[25] R. Hill, Proc. Phys. Soc. 65, 349 (1952).

[26] A. Yildirim, H. Koc, E. Deligoz, Chin. Phys. B 21, 37101 (2012).

[27] M.G. Brik, J. Phys. Chem. Solids 71, 1435 (2010).

[28] L. Shi, Y. Duan, X. Yang, G. Tang, Mater. Sci. Semicond. Process. 15, 499 (2012).

[29] H. Niu, X.Q. Chen, P. Liu, W. Xing, X. Cheng, D. Li, Y. Li, Sci. Rep. 2, 718 (2012).

[30] M. Jamal, N. Kamali, A. Yazdani, A.H. Reshak, $R S C$ Adv. 4, 57903 (2014).

[31] M. Born, K. Huang, Dynamical Theory of Crystal Lattices, Clarendon, Oxford 1954.

[32] J. Wang, S. Yip, S.R. Phillpot, D. Wolf, Phys. Rev. Lett. 71, 4182 (1993).

[33] D.C. Wallace, Thermodynamics of Crystals, Wiley, New York 1972

[34] B.B. Karki, G.J. Ackland, J. Crain, J. Phys. Condens. Matter. 9, 8579 (1997).

[35] G.A. Alers, J.R. Neighbours, J. Appl. Phys. 28, 1514 (1957).

[36] Shi Li-Wei, Duan Yi-Feng, Yang Xian-Qing, Tang Gang, Chin. Phys. Lett. 28, 100503 (2011).

[37] S.Q. Wang, H.Q. Ye, Phys. Status Solidi B 240, 45 (2003).

[38] L. Shi, Y. Duan, L. Qin, Comput. Mater. Sci. 50, 203 (2010).

[39] L. Kleinman, Phys. Rev. 128, 2614 (1962).

[40] http://en.wikiversity.org/wiki/Introduction_ to_Elasticity/Constitutive_relations.

[41] B. Pajot, B. Clerjaud, Optical Absorption of Impurities and Defects in Semiconducting Crystal, Springer, Berlin 2012, Ch. 8.

[42] H.M.A. Mazouz, A. Belabbes, A. Zaoui, M. Ferhat, Superlatt. Microstruct. 48, 560 (2010).

[43] F. Wooten, Optical Properties of Solid, Academic Press, New York 1972.

[44] C. Ambrosch-Draxl, J.O. Sofo, Comput. Phys. Commun. 175, 1 (2006).

[45] A. Delin, O. Eriksson, R. Ahuja, B. Johansson, M.S.S. Brooks, T. Gasche, S. Auluck, J.M. Wills, Phys. Rev. B 54, 1673 (1996).

[46] S.A. Korba, H. Meradji, S. Ghemid, B. Bouhafs, Comput. Mater. Sci. 44, 1265 (2009).

[47] Z.Y. Jiao, S.H. Ma, J.F. Yang, Solid State Sci. 13, 331 (2011).

[48] R. Goldhahn, A.T. Winzer, V. Cimalla, O. Ambacher, C. Cobet, W. Richter, N. Esser, J. Furthmüller, F. Bechstedt, H. Lu, W.J. Schaff, Superlatt. Microstruct. 36, 591 (2004).

[49] R. Goldhahn, P. Schely, M. Roppischer, Ellipsometry of InN and Related Alloys, CRC Press, 2007. 